\title{
artigo
}

\section{O uso da suplementação hiperproteica e creatina em pacientes idosos paliativos na cognição, funcionalidade e sarcopenia}

The use of hyperprotein and creatine supplementation in palliative elderly patients on cognition, functionality and sarcopenia El uso de suplementos de hiperproteína y creatina em pacientes ancianos paliativos em cognición, funcionalidad y sarcopenia

\section{RESUMO}

O envelhecimento é um processo natural, muitas vezes acometidos por doenças paliativas, o uso de suplementos orais pode melhorar fatores como sarcopenia, cognição e funcionalidade. Objetivo: Analisar o uso de suplementos hiperproteicos e creatina em pacientes idosos com doenças paliativas em relação a melhora da função cognitiva, funcionalidade e sarcopenia. Método: Trata-se de um artigo de revisão integrativo de literatura nas bases de dados PubMed, SciELO, Google Scholar, LILACS, no período de 2016-2020, utilizando os descritores do DeCS, envelhecimento, "cuidados paliativos", "suplementos nutricionais", "Idoso fragilizado", "disfunção cognitiva", sarcopenia e creatina, em idiomas português e inglês. Resultado: Foram encontrados 200420 artigos, após uma leitura criteriosa, apenas 62 artigos foram selecionados, sendo dividido em três seções mostrando os efeitos da suplementação hiperproteica e de creatina em idosos em paliativos. Conclusão: Mas estudos são necessários para comprovar o uso de suplementos em pacientes idosos paliativos na melhora da sarcopenia, funcionalidade e déficit cognitivo.

DESCRITORES: Cuidados Paliativos; Creatina; Sarcopenia.
\end{abstract}

\section{ABSTRACT}

Aging is a natural process, often affected by palliative diseases, the use of oral supplements can improve factors such as sarcopenia, cognition and functionality. Objective: To analyze the use of hyperprotein supplements and creatine in erderly patientswuth palliative diseases in relation to the improvement of cognitive function, functionality and sarcopenia. Methodo: This is na integrative literature review article in the PubMed, SciELO, Google Scholar, LILACS databases, in the period 2016-2020, using the DeCS descriptors, aging, "palliative care,"nutrtitional supplements", Elderly frail", "cogntive dysfunction", "sarcopenia and creatine, in Portuguese and English. Result: 200420 articles were found, after a careful Reading, only 62 articles were selected,being divided into three sections showing the effects of hyperprotein and creatine supplementation in palliative elderly. Conclusion: But studies are needed to prove the use of supplements in palliative eldery patients to improve sarcopenia, functionality and cogntive impairment.

DESCRIPTORS: Palliative Care; Creatine; Sarcopenia.

\section{RESUMEN}

El envejecimiento es un processo natural, muchas veces afectado por enfermedades por enfermidades paliativas, el uso de suplementos orales puede mejorar factores como la sarcopenia, la cognicion y la funcionalidade. Objetivo: Analizar el uso de suplementos de hiperproteínas y creatina en pacientes ancianos com enfermidades paliativas en relación con la mejora de la función cognitiva, la funcionalidade y la sarcopenia. Metodo: Se trata de un articulo de revisión integradora de la literatura em las bases de datos PubMed, SciELO, Google Scholar, LILACS, en el período 2016-2020, utilizando los descriptores DeCS, envejecimiento,"cudiados paliativos", suplementos nutricionales", ancianos frágiles', disfunción cognitiva", sarcopenia y creatina, en português e inglês. Resultado: 200420 artículos se encontraron, luego de uma lectura atenta, solo se seleccionaron 62 artículos, divididos em tres apartados que muestran los efectos de la suplementacion con hiperproteina y creatina em ancianos paliativos. Conclusión; Pero se necesitan estúdios para probar el uso de suplementos en pacientes ancianos paliativos para mejorar la sarcopenia, la funcionalidad y el deterioro cognitivo.

DESCRIPTORES: Cuidados paliativos; Creatina; Sarcopenia.

RECEBIDO EM: 11/01/2021 APROVADO EM: 16/02/2021 


\section{Marina de Oliveira Vieira}

Nutricionista e Educadora. Física - Orientadora de prática - UNICEUB. Mestranda em Ciências da Saúde - FEPECS. Especialista: Nutrição clínica, esportiva, fitoterápica, funcional oncologia e Cuidados paliativos - LABORO. Pós-Graduanda - Gerontologia e Assistência do Idoso.

ORCID: 0000-0002-5231-3231

\section{Ana Lúcia Ribeiro Salomón}

Nutricionista - Professora titular do UNICEUB, Orientadora de Mestrado da FEPCS. Doutora em ciências da saúde -UNB. Mestre em Nutrição Humana - UNB. Especialista em gestão em instituições de saúde - FEPECS. Especialista em Nutrição Parenteral e Enteral pela SBNPE. Especialista em Nutrição Clínica pela ASBRAN. Especialista em Nutrição Funcional pela VP/ UniCSUL. Especialista em Fitoterapia pela UNYLEYA.

ORCID: 0000-0002-1181-5948

\section{INTRODUÇÃO}

O Estatuto do Idoso, considera pessoas idosas aquelas com idade igual ou superior a 60 anos $^{1}$. O Brasil quando comparado a outros países como China, Índia, Estados Unidos e Indonésia sugere-se que em 2050, será a quinta maior população envelhecida do mundo, sendo necessário que esse envelhecimento ocorra de forma ativa ${ }^{2}$. A senescência é um processo natural que resulta na diminuição da capacidade funcional e atenuação dos mecanismos de defesa orgânicos, quando a reserva funcional do idoso é insuficiente, esse processo pode culminar no surgimento de senilidade demandando de Cuidados Paliativos $\left(\mathrm{CP}^{\prime} \mathrm{s}\right)^{3}$.

De acordo com o manual de CP's - Academia Nacional de Cuidados Paliativos. Os $\mathrm{CP}^{\prime} \mathrm{S}$ são abordagens que visam a promoção de qualidade de vida para pacientes e familiares que enfrentam doenças progressivas e ameaçadoras à vida ${ }^{4}$ Na literatura é possível observar que a maioria dos pacientes paliativos apresentam a sarcopenia ${ }^{5}$

A Sarcopenia em Pessoas Idosas é considerada uma doença muscular, resultando no aumento de riscos de quedas e fraturas o qual resulta em prejuízos nas atividades de vida diárias (AVD's) e nas atividades de vida instrumentais (AVI's). Tendo como componente central a perda de força, associada à diminuição de massa muscular ${ }^{6}$. Alguns suplementos orais podem ser benéficos para a melhoria da sarcopenia ${ }^{7}$. Em idosos sarcopênicos é necessário garantir um maior aporte de proteínas (PTN's) que podem atenuar essa resistência anabólica podendo ser de $1,2-1,5 \mathrm{~g} / \mathrm{kg} / \mathrm{dia}$ de $\mathrm{PTN}^{\prime} \mathrm{s}^{8}$. Muitos idosos não atingem a quantidade de PTN's necessárias. Os suplementos hiperproteicos podem diminuir os efeitos da sarcopenia em idosos ${ }^{9}$ sendo uma alternativa para garantir a quantidade mínima diária de PTN's na maioria dos $\operatorname{casos}^{10}$. De acordo com a diretriz da BRASPEN de terapia nutricional no envelhecimento é imprescindível o uso de suplementos orais em pacientes onde as necessidades nutricionais são maiores do que o gasto energético ${ }^{11}$.

A creatina é um outro suplemento utilizado - um composto de aminoácidos arginina, glicina e metionina, como uma boa alternativa em pacientes idosos com sarcopenia, melhorando a taxa de metabolismo proteico ${ }^{12}$. Pode ser usada na melhoria dos pacientes que sofreram traumas no Sistema Nervoso Central ou que têm doenças neurodegenerativas melhorando a cognição. ${ }^{13}$

O objetivo desse estudo é analisar na literatura científica, o uso de suplemento hiperproteico e creatina em pacientes idosos com doenças paliativas e em cuidados paliativos na melhora da função cognitiva, funcionalidade e sarcopenia.

\section{MÉTODOS}

Esse artigo consiste em uma revisão integrativa de literatura sobre o tema, de artigos publicados principalmente em revistas indexadas, nas bases de dados. Via base de dados da Medline (PubMed), Scientific Electronic Library Online (SciELO), Google Scholar, Latino Americano e do Caribe em Ciências da Saúde (LILACS), de 2016 a 2020, nos idiomas português, inglês e espanhol, utilizando os descritores cadastrados no DeCS: Envelhecimento; 'Cuidados paliativos", 'Suplementos nutricionais", funcional como "Idoso fragilizado", cognitivo como "disfun- ção cognitiva”, sarcopenia, creatina. Aplicando-se os conectores and e or e os seguintes descritores da saúde nas seções.

Os critérios de inclusão dos artigos foram baseados naqueles que atendiam a ideia proposta do tema, abrangendo a população idosa, o tempo escolhido para análise dos últimos cinco anos, com o uso de suplementação hiperproteica e creatina na melhora da sarcopenia, funcionalidade e cognição em idosos com enfermidades e cuidados paliativos, bem como artigos que visavam a importância dos cuidados nesse grupo com vulnerabilidade melhorando a qualidade de vida e diminuindo os custos do Sistema único de Saúde (SUS). Foram priorizados os artigos que não possuíam conflitos de interesses.

Foram excluídos os artigos que não se enquadravam no ano, nos descritores propostos assim como os temas, que não eram realizados ou que não se tratavam de idosos, nas seções de suplementos foram excluídos aqueles que não citavam os suplementos hiperproteicos ou de creatina. Para o propósito desse estudo foram considerados os pacientes em ( $\left.\mathrm{CP}^{\prime} \mathrm{s}\right)$ com ênfase em oncologia da descoberta até o tratamento e os diversos impactos na saúde, diminuindo a funcionalidade e gerando outros acometimentos como a sarcopenia que influi diretamente no estado nutricional e no sucesso do tratamento ${ }^{14}$, implicando também no impacto assistencial no SUS. De acordo com um estudo feito pelo observatório de oncologia, utilizando dados do ministério da saúde, Instituto Brasileiro de Geografia e Estatística ${ }^{5}$ o custo anual de 2016 em pacientes oncológicos pelo SUS chegou a aproximadamente 4 milhões de reais, sendo a maior parte desse em procedimentos clínicos e cirúrgicos.

A leitura completa resultou em 63 ar- 


\section{artigo}

Vieira, M.O.; Salomón, A.L.R.

O uso da suplementação hiperproteica e creatina em pacientes idosos paliativos na cognição, funcionalidade e sarcopenia

tigos, sendo esses 30 artigos do Google Scholar, 20 artigos do PubMed, 9 artigos da SciELO e 3 artigos no LILACS. Foram excluídos o total de 200353 artigos que não contemplavam as características exigidas.

\section{RESULTADOS}

Um total de 63 artigos foram utilizados para analisar os aspectos do envelhecimento, as doenças paliativas e a relação da suplementação oral de hiperproteico e creatina na melhora da sarcopenia, funcionalidade e cognição e os impactos no SUS. As políticas públicas também foram pesquisadas para compreendimento do contexto atual e como a alimentação pode ser crucial para aumentar a sua efetividade em idosos paliativos.

Foi dividido em três seções, a primeira seção relacionando o envelhecimento e as políticas públicas, a segunda seção subdivida em mais duas seções sobre as doenças e $\mathrm{CP}^{\prime}$ s, que atingem os idosos e sua qualidade de vida, fatores patológicos que influenciam na sarcopenia, cognição e funcionalidade e a suplementação hipeproteica e creatina na melhora dessas condições. E a terceira seção subdividida em mais duas seções sobre o estado nutricional em idosos paliativos e a relação da suplementação oral de hiperproteico concentrado e da creatina na sarcopenia, cognição e funcionalidade. Para a análise dos dados foi utilizado o método Prisma.

\section{DISCUSSÃO}

\section{0 impacto do envelhecimento e as políticas públicas para atenuar as causalidades}

A população idosa está crescendo de forma abrupta e como um cofator esse envelhecimento vem aliado a Doenças Crônicas Não Transmissíveis (DCNT's). Referente ao Brasil a estimativa é que a população idosa atinja $35 \%$ em $2070{ }^{16}$.

O envelhecimento pode gerar mudanças tanto no contexto social, fisiológico e biológico17. É importante manter e melhorar as atividades de vida diárias (AVD's) e as atividades de vida instrumentais (AVI's).

A figura 1. Representa o organograma no formato prisma da seleção de artigos, a partir da estratégia de busca descrita nos métodos.

Banco de dados PubMed, LILACS,

Google Scholar, SciELO 2016-2020

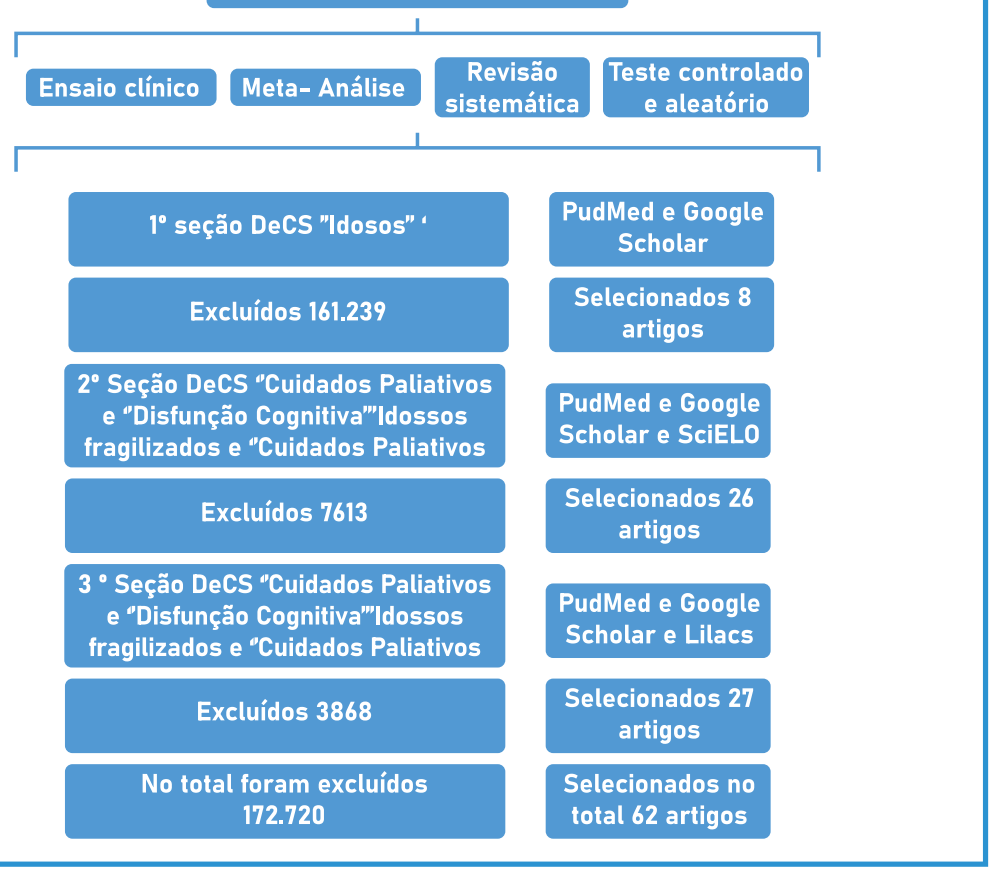

Junto com o envelhecimento alguns marcadores são alterados como uma maior concentração de tecidos adiposos abdominais e redução da massa muscular $(\mathrm{MM})^{18}$. Faz-se necessário tornar o envelhecimento ativo, melhorando a qualidade de vida funcional ${ }^{19}$.

As diversas alterações ocasionadas pelo envelhecimento necessitam da busca de um envelhecimento ativo, termo criado pela a OMS, para garantir a saúde, qualidade de vida, direitos, primazias e funcionalidade dos idosos ${ }^{20}$. Várias políticas foram criadas, para garantir este suporte ${ }^{21}$.O envelhecimento ativo deve apresentar um bem-estar geral, garantindo que esses idosos consigam realizar AVD's que são aquelas relacionadas ao autocuidado e as AVI's que exigem uma melhor consciência sobre a interação com o ambiente em que vive ${ }^{22}$.

Apesar de existirem vários programas com o intuito de melhorar a qualidade de vida dos idosos, ainda existem dificuldades no contexto assistencial. A Política Nacional de Saúde da Pessoa Idosa com o principal intuito de recuperar, manter e promover a saúde desse público reforça a importância e a necessidade de acompanhar a modo científico estratégias que possam melhorar o envelhecimento e diminuir as $\left(\mathrm{DCNT}^{\prime} \mathrm{S}\right)^{23}$. Com todas essas questões traçar estratégias que ajudem na saúde do idoso diminuindo os gastos do SUS, que aumentam com o avançar da idade, que poderão ser retornados em melhor suporte na saúde pública ${ }^{24}$.

Apesar das diversas perdas e déficits ocasionados a modo natural mediante ao processo de envelhecimento esse não significa que deve ser acometido por diversas doenças, porém o cenário atual é que muitos indivíduos estão passando por esse processo com vários efeitos deletérios, necessitando de cuidados entre eles $\mathrm{CP}^{\prime} \mathrm{s}^{25}$.

\section{Doenças Paliativas e Cuidados Palia- tivos em Idosos}

Estimasse que em 2030 o avanço da expectativa de vida, estará associado ao aumento de (DCNT's) que será resultante de $3 / 4$ de todas as mortes com ênfase, por cancros que necessitará de $\mathrm{CP}^{\prime} \mathrm{s}^{26}$. Quando a reserva funcional do idoso é insuficiente, esse processo pode culminar no surgimento de (senilidade) que 
podem demandar de $\left(\mathrm{CP}^{\prime} \mathrm{s}\right)^{27}$. O envelhecer traz a alteração da composição corporal podendo resultar na desnutrição, com a redução da massa muscular (MM) e de água intracelular e o aumento de tecido adiposo, gerando um aumento progressivo de peso até os 70 anos de idade e a partir de então, a redução de estímulos endócrino-metabólicos, inicia-se o processo de perda ponderal ${ }^{28}$.

De acordo com a (OMS), por ano $40 \mathrm{mi}-$ lhões de pessoas necessitam de CP's dessas $78 \%$ não possuem condições financeiras para realizar o tratamento a modo particular. $\mathrm{E}$ muitas políticas ainda não incluem os $\mathrm{CP}^{\prime}$ s, sendo essenciais a aplicabilidade no início das enfermidades ${ }^{29}$. Os $\mathrm{CP}^{\prime}$ 's visa o cuidado ao indivíduo, seu ambiente familiar e suas crenças, com o intuito de promover a melhora da qualidade de vida. ${ }^{30}$

No Brasil a maioria dos óbitos em idosos estão relacionados às (DCNT's) e aos cancros, com maior dependência do (SUS), já que as internações são mais longas do que quando comparadas a indivíduos jovens, um instrumento utilizado nos pacientes com doenças paliativas é a Palliative Performance Scale (PPS), após a sua aplicação é possível ter uma concepção sobre a integridade, conforto, dignidade e qualidade de vida do paciente ${ }^{31}$. A PPS pode ser usada também para indicar os idosos que necessitam de CP's e a evolução do diagnóstico do paciente ${ }^{32}$.

Doença Paliativa é toda doença crônica que não apresenta cura, as mais prevalentes em idosos são: as oncológicas, as que geram danos neurológicos com prognóstico ruim, Alzheimer, demências, Doença Pulmonar Crônica Obstrutiva (DPOC), Parkinson, tumores cerebrais ${ }^{33}$. A maioria dos pacientes geriátricos com doenças paliativas apresentam várias demandas, sendo de suma importância que a equipe domiciliar compreenda as necessidades dos pacientes ${ }^{34}$. Uma das funções que apresentam um grande impacto na vida desses senis é a cognição, gerando uma queda da qualidade de ${ }^{35}$.

\section{A cognição no envelhecimento asso- ciado a doenças paliativas}

Com o avanço da idade, um dos acometimentos é o declínio da cognitivo ${ }^{36}$. É vital que os profissionais da saúde entendam que os $\mathrm{CP}^{\prime} \mathrm{s}$ não devem ser limitados apenas para pacientes no fim da vida, alguns fatores em pacientes paliativos como os cognitivos muitas vezes não são avaliados, de acordo com um estudo realizado com 70 pacientes em uma unidade geriátrica, apenas $11 \%$ tinham ciência da sua doençą ${ }^{37}$.

$O$ fator cognitivo é afetado em pacientes paliativos com neoplasias malignas, ocorrendo um declínio nos domínios cognitivos, podendo estar relacionado ao tratamento com o aumento das citocinas pró inflamatórias podendo relacionar de modo direto à cognição ${ }^{38}$.

Um dos protocolos clínicos para a avaliação de idosos na disfunção cognitiva é o Mini Exame do Estado Mental (MEEM), aceito mundialmente, podendo avaliar o grau de demência, resposta a tratamento sugeridos na melhora da cognição, e o nível de dificuldade para aplicação é baixo ${ }^{39}$. As disfunções cognitivas leves presentes em doenças paliativas como no câncer em estágio avançado, pode estar relacionada ao tratamento, os profissionais da área da saúde devem se manterem atentos a todos os sinais ${ }^{40}$.

Um estudo analisando 87 pacientes idosos com cancros, através do MEEM, mostrou que $29 \%$ dos pacientes apresentavam declínio cognitivo, mais presente no gênero feminino e que esse regresso cognitivo pode gerar uma má qualidade de vida para esses pacientes que também podem apresentar declínio funcional ${ }^{41}$.

\section{Declínio Funcional em Idosos acometi- dos por doenças paliativas}

O declínio funcional, pode ocorrer de maneira natural como processo de senilidade sendo agravado quando associado a doenças paliativas $^{42}$. A capacidade funcional oferece aos indivíduos independência, propiciando executar suas atividades diárias e instrumentais. Um dos instrumentos utilizados é a escala de Kartz; um estudo avaliando 150 pacientes idosos com a maioria do gênero masculino atendidos no ambiente domiciliar mostrou que ações intervencionais na melhora da recuperação funcional pode melhorar a qualidade de vida de paciente paliativos ${ }^{43}$.A resistência anabólica é muito presente em pacientes idosos, resultando na degradação da Massa Muscular (MM) e diminuição de força ${ }^{44}$.
Um outro estudo avaliando 94 indivíduos, em CP's, com aproximadamente 60 anos, no Brasil, com câncer avançado, com a maior prevalência do gênero feminino, sendo os cancros de maior representatividade entre os grupos os gastrointestinais, de mama e pulmão mostrou que $40 \%$ apresentavam um declínio funcional ${ }^{45}$. Em pacientes oncológicos em CP's manter a capacidade funcional é fundamental já que o seu declínio pode gerar piora do prognóstico ${ }^{46}$.

Um outro estudo realizado na atenção primária em idosos indicados para cuidados paliativos, com uma amostragem de 73 indivíduos, sendo o gênero feminino predominante apresentou que quando comparado duas doenças como o câncer e doenças neurológicas, as quais apresentaram uma maior diminuição da capacidade funcional, entretanto em ambos os grupos se via alguma limitação funcional ${ }^{47}$. A baixa funcionalidade vista em pacientes em CP's apresenta relação com os fatores nutricionais como a caquexia, piorando o prognóstico do paciente levando a diminuição de (MM) e também tecido adiposo visto em pacientes oncológicos ${ }^{48}$. Outro fator que pode estar associado a diminuição da funcionalidade e a qualidade de vida dos pacientes oncológicos é a sarcopenia, geralmente ocasionada pelo próprio tratamento ${ }^{49}$.

\section{Estado nutricional e Sarcopenia em Paliativos}

A sarcopenia conhecida pela diminuição da (MM) e da força, frequente em idosos; encontra-se de forma mais acentuada, quando associada a uma doença entre elas as paliativas; à baixa ingesta de PTN's, distúrbios gastrointestinais, iatrogenias, anorexia. Além disso contamos com um maior tempo de hospitalização, maior tempo de imobilização (contensão ao leito ou mínima deambulação), o que implica menor qualidade de vida, maiores custos para o SUS e uma maior incapacidade funciona ${ }^{50}$. A alimentação em $\mathrm{CP}^{\prime} \mathrm{s}$ deve ocorrer de maneira individualizada ${ }^{51}$. A avaliação nutricional deve ser parte dos cuidados geriátricos principalmente quando apresentam comorbidades, podendo avançar para a sarcopenia aumentando os índices de mortalidade ${ }^{52}$. Indivíduos sarcopênicos apresentam um maior declínio de MM gerando 


\section{artigo}

danos funcionais, principalmente nos membros inferiores ${ }^{53}$. A maioria dos pacientes paliativos oncológicos apresentam disfunções no estado nutricional levando em vários casos a desnutrição e resultando na diminuição da MM e da força que pode ser mensurada através da força de preensão manual ${ }^{54}$.

Os tratamentos oncológicos quimioterápicos e radioterápicos apresentam vários efeitos deletérios, levando a fadiga que diminui a qualidade de vida dos pacientes, ocasionando alterações nutricionais que resulta na sarcopenia ${ }^{55}$. Em um estudo avaliando o estado nutricional e a relação da perda de peso no período de seis meses, em um público com a idade média de 63 anos, com 104 indivíduos, com a maioria do gênero masculino, em CP's com predominância de adenocarcinoma concluiu que é um fator que deve ser levado em consideração desde os últimos três meses ${ }^{56}$.

Uma pesquisa realizada com 70 pacientes, a maioria do gênero masculino, com tumores mais prevalentes de cabeça e pescoço, com a via alimentar prevalente oral com uso de suplementação, mostrou que $87,2 \%$ possuíam desnutrição, resultando na diminuição de força física dos pacientes ${ }^{57}$.

$\mathrm{O}$ catabolismo visto em pacientes paliativos oncológicos associado a uma má nutrição, leva a depleção da (MM) e déficit de força, sendo importante uma intervenção nutricional, o uso de suplementações podem apresentar um efeito benéfico nesses pacientes ${ }^{58}$.

\section{Uso de suplementos orais hiperprotei- cos e na melhora da sarcopenia, fun- cionalidade e cognição em paliativos}

Entre os CP's a alimentação deve ser um dos fatores de grande relevância durante o tratamento podendo amenizar o sofrimento do paciente, o uso de suplementos por via oral é indicado quando os pacientes não conseguem atingir a demanda energética ${ }^{59}$, sendo uma das condutas de maior aceitação na maioria dos casos sem restrições, contribuindo para o suporte energético adequado, melhorando a ingestão proteica principalmente em pacientes em tratamento de cancros ${ }^{60}$. De acordo com o consenso do Guideline da European Society for Clinical Nutrition and Metabolism (ESPEN) a suplementação oral em pacientes paliativos com doenças avançadas é uma das melhores opções ${ }^{61}$.

Em um estudo visando a qualidade de vida em pacientes mulheres em $\mathrm{CP}^{\prime}$ 's com diversos cancros, na Índia com total de 69 mulheres, com caquexia, quando associado a suplementação entre elas hiperproteica pode melhorar a qualidade de vida das pacientes assim como ajudar na manutenção do peso, os hiperproteicos utilizados foram a base de algumas farinhas e a cada $400 \mathrm{~g}$, $50 \%$ era de PTN ${ }^{62}$.

A adição de fórmulas suplementares hiperproteicas e hipercalóricas apresentam vários benefícios quando adicionadas a condutas dietoterápicas, de acordo com a orientação da (ESPEN), em pacientes ambulatoriais ou em situações que necessitem de apoio nutricional, sem intolerância as PTN's intactas podem ser usadas no tratamento ${ }^{63}$.

A Força Muscular (FM) e a função física podem ser beneficiadas melhorando a resposta anabólica quando a ingestão de suplementação proteica é desmembrada no período do desjejum com $15 \mathrm{~g}$ e no período pós-prandial com $15 \mathrm{~g}$ melhorando a resposta anabólica ${ }^{64}$.

Os suplementos proteicos são benéficos na melhora dos acometimentos ocorridos pelo próprio envelhecimento entre eles a sarcopenia, na geriatria é necessário um aporte maior de PTN's, muitas vezes não sendo atingidos pelos idosos, sendo sugerido o uso de 1,2 a $1,5 \mathrm{~g} / \mathrm{kg} /$ dia, principalmente a partir de 71 anos ${ }^{65}$.

Os pacientes sarcopênicos com Doenças Renais Crônicas (DRC), devem ter uma limitação diária de PTN's ${ }^{66}$ Apesar da necessidade de PTN que muitos idosos precisam para a melhora da sarcopenia, é necessário um cuidado no consumo em pacientes que apresentem uma taxa glomerular inferior a $30 \mathrm{ml} / \mathrm{min}^{67}$.

\section{Suplementação oral de creatina na sar- copenia, funcionalidade e cognição}

A creatina é um composto de aminoácido arginina, glicina e metionina, o próprio envelhecimento reduz a produção de creatina, entretanto a suplementação exógena de creatina, pode melhorar esses estoques entre quatro a seis semanas principalmente quando associado a atividade física ${ }^{68}$. Recomenda-se de 3 à $5 \mathrm{~g}$ diários para a manutenção de níveis elevados de creatina ${ }^{69}$. A creatina é atualmente sendo um dos suplementos de grande desta- que na sarcopenia ${ }^{70}$, sendo considera como um suplemento completo ${ }^{71}$.

A sarcopenia é vista em maior predominância em pacientes acima de 60 anos, atingindo a funcionalidade e diminuindo a qualidade de vida, degradação muscular, resultante da resistência anabólica, o uso da suplementação oral de creatina pode ser benéfica em pacientes idosos na fadiga, na MM, no desempenho das (AVD's) e cognição, na sarcopenia ela apresenta melhora na fadiga, sendo recomendado o uso de creatina em idosos em déficit ou falta da mesma, devendo ser associada com carboidratos e não indicada para pacientes com $\mathrm{DRC}^{72}$.

Um estudo duplo cego, realizado em idosos, com diferentes níveis de sarcopenia, pouco ativos, do gênero masculino, sendo selecionados 45 homens e finalizando o estudo com 32 indivíduos, em um período de 12 semanas com o uso de $3 \mathrm{~g}$ de creatina assim como de outros suplementos alimentares, com recomendação de atividade física de baixa intensidade, melhorou a MM, o desempenho em homens fisicamente inativos ${ }^{73}$.

A suplementação de creatina, tem sido vista como uma alternativa na melhora da cognição, atuando de forma neuroprotetora melhorando os níveis de energia cerebral beneficiando principalmente o público idoso ${ }^{74}$. Podendo exercer um papel nos quadros depressivos de pacientes, um estudo realizado entre indivíduos de 19 a 65 anos mostrou que pode colaborar na melhora energética cerebral, melhorando a receptação de serotonina, por oito semanas, sendo na primeira semana de $3 g$ e nas outras sete semanas de $5 \mathrm{~g}^{75}$.

Um outro estudo avaliando a suplementação de creatina e de outro suplemento em 108 pacientes em ambiente hospitalar, na Itália, de ambos os gêneros com idades entre 60 a 85 anos mostrou que a creatina pode melhorar a capacidade funcional, em pacientes com (DPOC) que se encontravam estáveis como também na disfunção de apnéia ${ }^{76}$.

A suplementação de creatina, pode aumentar os estoques de energia intramuscular, em pacientes com cancro de próstata onde a uma diminuição de (MM) um estudo com 56 indivíduos, com a doença avançada, com idade de 65 anos ou menos, mostrou que pode apresentar efeitos benéficos, porém mais estudos preci- 
sam ser feitos para que mostre a real eficácia da creatina em idosos acometidos com câncer ${ }^{77}$.

\section{CONCLUSÃO}

O envelhecimento faz parte do processo natural da vida, ocorrendo o declínio de diversas funções gerando danos celulares , principalmente quando associados a doenças paliativas necessitando de medidas que melhorem fatores decorrentes dessas enfermidades.A suplementação de hiperproteico e de creatina podem ser benéficas, atenuando a sarcopenia a funcionalidade e o cognitivo. Sendo imprescindível que os profissionais da saúde estejam atentos a esses fatores podendo melhorar a qualidade de vida desses pacientes restaurando a condição nutricional, melhorando o prognóstico, diminuindo o tempo de hospitalização. Mas estudos devem serem feitos tendo como base a suplementação oral de creatina e hiperproteico na melhora da sarcopenia, funcionalidade e cognitivo em pacientes idosos paliativos.

\section{REFERÊNCIAS}

1. Dispõe sobre o Estatuto do Idoso e dá outras providências [Internet]. Brasilia: LUIZ INÁCIO LULA DA SILVA; 2003 Oct 01. LUIZ INÁCIO LULA DA SILVA; [cited 2020 Mar 18]; Available from: http:/www.planalto. gov.br/ccivil_03/leis /2003/l10.741.htm

2. Oliveira FM, et al. Síndrome do idoso frágil: análise conceitual de acordo com Walker e Avant. Rev. Bras. Enferm [Internet]. 2020 Mar 13 [cited 2020 Feb 20];73(3):1-8. DOI https:/doi.org/10.1590/00347167-2019-0601. Available from: https:/www. scielo.br/scielo. php?pid=S0034-71672020001500302\&script =sci_arttext\&tlng=pt

3. Confortin S.C, et al. Sarcopenia e sua associação com mudanças nos fatores socioeconômicos, comportamentais e de saúde: Estudo EpiFloripa Idoso. CSP- Cadernos de Saúde Pública, Santa Catarina- Florianópolis, 2018.

4. Manual de Cuidados Paliativos: ANCP. 2nd rev. ed. São Paulo: ANCP; 2012. 590 p. 2 vol.

5. QUEIROZ M, et al. Associação entre Sarcopenia, Estado Nutricional e Qualidade de Vida em Pacientes com Câncer Avançado em Cuidados Paliativos: Sarcopenia: Revista Brasileira de Cancerologia, Rio de Janeiro, v. 64, ed. 1, p. 69-75, 2018.

6. Jentoft A. Sarcopenia: revised European consensus on definition and diagnosis. GUIDELINES [Internet]. 2018 Oct 12 [cited 2020 Jul 1];1(48):1-16. DOI 10.1093/ageing/afy169. Available from: https:/ www.ncbi.nlm.nih.gov/pubmed /30312372

7. Chan MY, Chok KSH. Sarcopenia in pancreatic cancer - effects on surgical outcomes and chemotherapy. World J Gastrointest Oncol [Internet]. 2019 Jul 15 [cited 2020 Oct 1];11(7):522-537. DOI 10.4251/wjgo.v11.i7.527. Available from: https:/pubmed.ncbi.nlm.nih. gov/31367272/.

8. Teshima C, et al. Suplementação de Aminoácidos: Influência na Sarcopenia em Idosos - uma Breve Revisão. International Journal of Nutrology, Rio de Janeiro, p. 1-11, 2018. DOI 10.1055/s-0038-1675030. Disponivel em: https:/www.thieme-connect.com/products/ejournals /html/10.1055/s-0038-1675030. Acesso em: 28 dez. 2019.

9. Liberman, K. et al. Thirteen weeks of supplementation of vitamin D and leucine-enriched whey protein nutritional supplement attenuates chronic low-grade infammation in sarcopenic older adults: the provide study. Aging Clinical and Experimental Research, [S. I.], 2 maio 2019. Disponível em: (https://doi.org/10.1007/s40520-019-01208-4). Acesso em: 4 maio 2019.

10. Volkert D. et al. Espen guideline on clinical nutrition and hydration in geriatrics. Clinical Nutrition, European, p. 10-47, 28 fev. 2019. http:/ www. elsevier. com /locate/clnu.
11. Gonçalves Thiago José Martins, et al. Diretriz BRASPEN de terapia nutricional no envelhecimento. Brazilian Society of Parenteral and Enteral Nutrition: BRASPEN. 2019;34(3):2-58.

12. Somoza EZ, et al. Sobre las interrelaciones entre la sarcopenia, el envejecimiento y la nutrición. Revista Cubana de Alimentación y Nutrición. 2018;28(1):152-176.

13. Patier PHX, et al. Pacientes em Suplementação de Creatina Monohidratada Frente a Aspectos da Neuroproteção Cerebral. Thieme Revinter , Rio de Janeiro, p. 1-37, 6 maio 2018.

14. Cordeiro Antonia Lucas de Oliveira, Fortes Renata Costa. Estado nutricional e necessidade de intervenção nutricional em mulheres com câncer de mama em tratamento quimioterápico. Arquivos Catarinenses de Medicina: AMB. 2015 Outubro;44(4):96-108.

15. Gastos federais em oncologia [Internet]. São Paulo: Tiago Cepas; 2018 Jan 01. Gastos Federais em Oncologia; [revised 2020 Jun 25; cited 2020 Jun 25]; Available from: https://observatoriodeoncologia.com. br/gastos-federais-em-oncologia/.

16. Santos SL, et al. Envelhecimento populacional e gastos com saúde: uma análise das transferências intergeracionais e intrageracionais na saúde suplementar brasileira. Revista Brasileira de Estudos de População, Belo - Horizonte, v. 35, ed. 2, p. 1-30, 25 fev. 2019. DOI https://doi. org/10.20947/s102-3098a0062. Disponivel em: https:/www.scielo. br/scielo.php?script=sci _arttext\&pid=S0102-30982018000200155. Acesso em: 28 ago. 2020.

17. Souza TFC, et al, Prevenção da Sarcopenia em Idosos. Caderno de Educação Saúde e Fisioterapia; São Mateus - ES; volume 05. N¹0; 2018.

18. Brandão BM, et al. Relação da cognição e qualidade de vida entre idosos comunitários: estudo transversal. Rev. Bras. Enferm. , Brasilia, v. 73, ed. 3, p. 1-7, 8 jul. 2020. DOl https://doi.org/10.1590/0034-71672019-0030. Disponivel em: https:/www.scielo.br/pdf/reben/v73s3/ pt_0034-7167-reben-73-s3-e20190030.pdf. Acesso em: 26 ago. 2020.

19. Colussi EL, et al. Percepções de idosos sobre envelhecimento e violência nas relações intrafamiliares. Rev. bras. geriatr. gerontol., Rio de Janeiro, v. 22, ed. 4, p. 1-8, 24 out. 2019. DOl https:/ doi.org/10.1590/1981-22562019022.190034. Disponivel em: https:/www.scielo.br/pdf/rbg g/v22n4/pt_1809-9823-rbgg-2204-e190034.pdf. Acesso em: 26 ago. 2020.

20. Wagner $\mathrm{KH}$, et al. Biomarkers of Aging: From Function to Molecular Biology. Nutrients., [s. I.], v. 8, ed. 338, p. 1-12, 30 maio 2016. DOI doi:10.3390/nu8060338. Disponivel em: https:/www.nc bi.nlm.nih. 


\section{REFERÊNCIAS}

gov /pmc/articles/PMC 4924179/pdf/nutrients-08-00338.pdf. Acesso em: 26 ago. 2020.

21. Leite ES, et al. Tecnologia assistiva e envelhecimento ativo segundo profissionais atuantes em grupos de convivência. Revista da Escola de Enfermagem da USP, São Paulo, 13 set. 2018. http://dx.doi. org/10.1590/s1980-220×2017030903355.

22. Rantanen T. et al. Active aging - resilience and external support as modifiers of the disablement outcome: AGNES cohort study protocol. BMC Public Health, Finland, v. 18, ed. 565, p. 1-21, 2 maio 2018. DOI 10.1186/s12889-018-5487-5. Disponivel em: https:/www .ncbi. nl m.nih.gov/pmc/a rticles/PMC5930766/pdf/1288 9_2018_Article_5487.pdf. Acesso em: 26 ago. 2020.

23. Lange C, et al. Promoção da autonomia de idosos rurais no envelhecimento ativo. Revista Brasileira de Enfermagem, Brasilia, v. 71, ed. 5, p. 2555-2561, outubro 2018. DOI http://dx.doi.org/10.1590/00347167-2017-0570. Disponível em: https:/www.scielo.br/pdf/reben/ v71n5/pt_0034-7167-reben-71-05-2411.pdf. Acesso em: 27 ago. 2020.

24. Sciama DS, et al. Envelhecimento ativo: representações sociais dos profissionais de saúde das Unidades de Referência à Saúde do Idoso*. Revista da Escola de Enfermagem da USP [Internet]. 2019 Sep 07 [cited 2020 Jul 30];54(e03605):1-10. DOI https://doi.org/10.1590/ s1980-220x2018056503605. Available from: https:/www.scielo.br/ pdf/reeusp/v54/1980-220X-reeusp-54-e03605.pdf

25. Labegalini CMG, et al. Caminho dialógico educacional e relacionado ao cuidado sobre o envelhecimento ativo com profissionais de estratégia de saúde da familia. Texto \& Contexto - Enfermagem, Paraná, v. 29, p. 1-14, 20 maio 2020. DOI http://dx.doi.or g/10.1590/19 80-265x-tce-2018-0235. Disponivel em: http:/www.revenf.bvs. br/scielo.php?script=sci_arttext\&pid=S01040707202000100335. Acesso em: 28 ago. 2020.

26. Santos SL, et al. Envelhecimento populacional e gastos com saúde: uma análise das transferências intergeracionais e intrageracionais na saúde suplementar brasileira. Revista Brasileira de Estudos de População, Belo - Horizonte, v. 35, ed. 2, p. 1-30, 25 fev. 2019. DOI https://doi. org/10.20947/s102-3098a0062. Disponivel em: https:/www.scielo. br/sci elo.php?script=sci _arttext\&pi d=S0102-30982018 00020015 5. Acesso em: 28 ago. $20 \overline{20}$.

27. Pereira BR, et al, Perfil sociodemográfico da mortalidade da população idosa no nordeste brasileiro. Revista de Atenção á Saúde: RAS, Salvador, v. 18, ed. 64, p. 9-21, 5 fev. 2020. DOl https//doi. org/10.13037/ras.vol18n64.6273. Disponivel em: https:/seer.uscs. ed u.br/index.php/revista _ciencias_saude/article/ view/62 73/pdf. Acesso em: 28 ago. 2020.

28. Oliveira LC, Cuidados Paliativos: Por que Precisamos Falar sobre isso?. Revista Brasileira de Cancerologia, Rio de Janeiro, v. 65, ed. 4, p. 1-3, 2019. DOl https:/doi.org/10.32635/2176-9745. RBC.2019v65n4.558. Disponivel em: https:/rbc.in ca.gov.br/revista/i ndex.php/revista/article/ view/558/499. Acesso em: 3 set. 2020.

29. Maneira A, Zanata IL. A frequência de disfagia em idosos em um hospital da cidade de curitiba .PR. Revista de Saúde Pública do Paraná. 2018julho;1(1):20-26.
30. Ohashi K, et al. Relationship Between Sarcopenia and Both Physical Activity and Lifestyle in Patients With Chronic Liver Disease. Journal of clinical medicine research, v. 10, n. 12, p. 920, 2018.

31. Gontijo S, et al. Envelhecimento ativo: conceito e fundamento: 0 que é "envelhecimento ativo" OMS. In: Envelhecimento ativo: uma política de saúde. 1. 1². ed. Brasilia: ENVELHECIMENTO ATIVO: UMA POLITICA DE SAÚDE, 2005. Disponivel em: http:/bvsms.saude.gov.br/ bvs/publica coes/envelhecimento_ativo.pdf. Acesso em: 27 ago. 2020.

32. Gouvea MPG. A necessidade de cuidados paliativos para paciente com doenças crônicas: diagnóstico situacional em um hospital universitário. Revista Brasileira de Geriatria e Gerontologia: Rev. bras. geriatr. gerontol, Rio de Janeiro, v. 22, ed. 5, p. 1-9, 3 fev. 2020. DOI http://dx.doi.org/10.1590/1981-22562019022.190085. Disponível em: https:/www.scielo.br/pdf/rbgg/v22n5/pt_1809-9823-rbgg-2205-e190085.pdf. Acesso em: 3 set. 2020.

33. Clara MGS, et al. Escala Palliative Care Screening Tool como instrumento para indicação de cuidados paliativos em idosos. Rev. Bras. Geriatr. Gerontol., Espirito Santo, v. 22, ed. 5, p. 1-10, 28 nov. 2019. DOI http://dx.doi.org/10.1590/1981-22562019022.190045. Disponivel em: https:/rbgg.com.br/mobile/edicoes/v22n5/RBGG\%20v22n5\%20 PORT_2019-0143.pdf. Acesso em: 3 set. 2020.

34. Robinson MT; Holloway, RG, Palliative Care in Neurology. Mayo Clin Proc .: Elsevier, [s. I.], v. 92, ed. 10, p. 1592-1601, Outubro 2017. DOI 10.1016/j.mayocp.2017.08.003. Disponivel em: https:/pubmed.ncbi. nlm.nih.gov/28982489/. Acesso em: 20 set. 2020.

35. Sydney MDY, et al. Palliative Care for Cancer Survivors. Med Clin North: HHS Public Access, [s. I.], v. 101, ed. 6, p. 1181-1196, 2017. DOI 10.1016/j.mcna.2017.06.009. Disponivel em: https://referenciabibliografica.net/a/pt-br/ref/abnt. Acesso em: 20 set. 2020.

36. Voumart $\mathrm{R}_{1}$. et al. Geriatric palliative care: a view of its concept, challenges and strategies. BMC Geriatrics, EUA, v. 18, ed. 220, p. 1-6, 20 set. 2018. DOI 10.1186 / s12877-018-0914-0. Disponível em: https:/www.ncbi.nlm.nih.gov/pmc/articles/PMC6148954/ pdf/12877_2018_Article_914.pdf. Acesso em: 20 set. 2020.

37. Freire MEM, et al. Qualidade de vida relacionada à saúde de pacientes com câncer em cuidados paliativos. Texto contexto, [s. I.], v. 27, ed. 2, p. 1-13, 28 maio 2018. DOl https://doi.org/10.1590/0104070720180005420016. Disponivel em: https:/www.scielo.br/pdf/ tce/v27n2/0104-0707-tce-27-02-e5420016.pdf. Acesso em: 24 set. 2020.

38. Arcanjo SP, et al. Características clínicas e laboratoriais associadas à indicação de cuidados paliativos em idosos hospitalizados. EINSTEN, São Paulo, v. 16, ed. 1, p. 1-8, 2018. DOI DOI: 10.1590/ S1679-45082018A04092. Disponível em: https://journal.einstein. br/wp-content/uploads/articles_xml/1679-4508-eins-16-01eA04092/1679-4508-eins-16-01-eA04092-pt.x57660.pdf. Acesso em: 24 set. 2020.

39. Lima CVC; Póvoa , RMF. Mulheres Submetidas à Quimioterapia e suas Funções Cognitivas. Psicologia: Ciência e Profissão, [s. I.], v. 37, ed. 4, p. 970-980, 2017. DOI https://doi.org/10.1590/19823703004772016 Mulheres Submetidas à. Disponivel em: https:/ www.scielo.br/pdf/pcp/v37n4/1414-9893-pcp-37-04-0970.pdf. Acesso em: 20 ago. 2020. 
40. Sousa NFS, et al. Envelhecimento ativo: prevalência e diferenças de gênero e idade em estudo de base populacional. Cadernos de saúde pública: csp, Cuiabá, v. 34, ed. 11, p. 1-16, 23 nov. 2018. DOI http://dx.doi. org/10.1590/0102-311x00173317. Disponivel em: https:/ww w.scielo.br/sci elo.php? script=sci _artte xt\&pid=S0102-311X201800110 5007\&lng =en\&nrm=iso\&tlng=en. Acesso em: 26 ago. 2020.

41. Sato ACT, et al. Estado mental dos cuidadores de idosos em hemodiálise. REFACS, Macapá, v. 8, ed. 3, p. 1-9, 1 jul. 2020. DOI 10.18554/refacs.v8i3.3932. Disponível em: http:/seer.uftm.edu.br/ revistaeletronica/index.php/refacs/article/view/3932/pdf. Acesso em: 27 set. 2020.

42. Kurita K, et al. Associações entre Disfunção Cognitiva Leve e Resultados de Fim de Vida em Pacientes com Câncer Avançado. Journal of Palliative Medicine, [s. I.], v. 21, ed. 4, p. 536-540, 1 abr. 2018. DOI 10.1089/.jpm.2017.0385. Disponivel em: https:/pubmed.ncbi.nlm.nih. gov/29298104/. Acesso em: 27 set. 2020.

43. Akechi T, et al. O declínio cognitivo diminui o valor da utilidade para a saúde em pacientes idosos com câncer?. Psicogeriatria , [s. I.], v. 17, ed. 3, p. 149-154, 13 jul. 2016. DOI 10.1111 / psyg.12205. Disponível em: https:/pubmed.ncbi.nlm.nih.gov/27411897/. Acesso em: 27 set. 2020.

44. Gaspar RB, et al. Fatores condicionantes para o enfermeiro defender a autonomia do idoso na terminalidade da vida. Revista Brasileira de Enfermagem, Brasilia, v. 73, ed. 3, p. 1-8, 13 jul. 2020. DOI https://doi. org/10.1590/0034-7167-2018-0857. Disponivel em: https:/www. scielo.br/scielo.php?script=sci_arttext\&pid=S0034-7167202000150 0158\&lang=pt. Acesso em: 27 set. 2020.

45. Cerezer LG, et al. Avaliação da (Evaluación de la) capacidade funcional de pacientes em atenção domiciliar. Salud ciencia, Brasil, Santa Maria, v. 22, p. 525-531, 24 fev. 2017. DOI http://dx.doi.org/10.21840/ siic/150394. Disponível em: https:/www.siicsalud.com/dato/ sic/226/150394.pdf. Acesso em: 27 set. 2020.

46. Nassr GNM, Atrofia muscular esquelética: relação entre ciências básicas e aplicadas. Fisioterapia e Pesquisa, Chile, 2019. http://dx.doi.or g/10.1590/1809- 2950/00000026012019.

47. Pereira MME, etal. Ângulo de Fasee Estado Nutricional em Indivíduos com Câncer Avançado em Cuidados Paliativos. Revista Brasileira de Cancerologia [Internet]. 2019 Jun 06 [cited 2020 Sep 15];65(1):16. DOI https://doi.org/10.32635/2176-9745.RBC.2019v65n1.272. Available from: https://rbc.inca.gov.br/revista/index.php/revista/article/ view/272/181

48. Scottini MA; Moritz RD; Siqueira JE, Cognition, functionality and symptoms in patients under home palliative care. Revista da Associação Médica Brasileira, São Paulo, Brasil, v. 64, ed. 10, p. 922-927, outubro 2018. DOI https:/doi.org/10.1590/1806-9282.64.10.922. Disponivel em: https:/pubmed.ncbi.nlm.nih.gov/30517240/. Acesso em: 27 set. 2020.

49. Marcucci FCl, et al. Capacidade funcional de pacientes com indicação de cuidados paliativos na atenção primária. Geriatrics Gerontology and aging, Londrina, Brasil, v. 12, ed. 3, p. 159-165, 2018. DOI 10.5327/ Z2447-211520181800026. Disponivel em: http:/www.ggaging.com/ details/482/pt-BR/functional-capacity-of-patients-indicated-for-palliative-care-in-primary-care. Acesso em: 27 set. 2020.
50. Davis MP, Panikkar R, Sarcopenia associated with chemotherapy and targeted agents for cancer therapy. Annals of Palliative Medicine, USA, v.8, ed. 1, p.86-101, 2ago. 2018. DOI 10.21037/apm.2018.08.02. Disponivel em: https:/pubmed.ncbi.nlm.nih.gov/30525762/. Acesso em: 27 set. 2020.

51. Queiroz M, et al. Associação entre Sarcopenia, Estado Nutricional e Qualidade de Vida em Pacientes com Câncer Avançado em Cuidados Paliativos: Sarcopenia: Revista Brasileira de Cancerologia, Rio de Janeiro, v. 64, ed. 1, p. 69-75, 2018.

52. Benarroz MO, et al. Anorexia: an Eating Challenge in Palliative Care. Revista Brasileira de Cancerologia , Rio de Janeiro, Brasil, v. 65, ed. 2, p. 1-3, 2019. DOl https://doi.org/10.32635/2176-9745. RBC.2019v65n2.98. Disponível em: https://rbc.inca.gov.br/revista/index.php/revista/article/view/98. Acesso em: 30 set. 2020.

53. Jentoft AJC, Sarcopenia: what should a pharmacist know?. Farmacia Hospitalaria, Madrid, v. 41, ed. 41, p. 543-549, 5 abr. 2017. DOI DOI: 10.7399/fh.2017.41.4.10802. Disponivel em: https:/www.sefh.es/ fh/161_11revision0110802.pdf. Acesso em: 1 out. 2020.

54. Souza BLB, MFS.Os transtornos neuropsicológicos e cognitivos da doença de Alzheimer: a psicoterapia e a reabilitação neuropsicológica como tratamentos alternativos. Pretextos, [s. I.], v. 3, ed. 5, p. 467-484, 7 mar. 2018.

55. Silva, EHE, et al. Associação entre Estado Nutricional e Força de Preensão Manual em Pacientes Oncológicos em Cuidados Paliativos. Revista Brasileira de Cancerologia, Minas Gerais, Brasil, v. 64, ed. 4, p. 479-487, 2018. DOI https:/doi.org/10.32635/2176-9745. RBC.2018v64n4.196. Disponivel em: https://rbc.inca.gov.br/revista/ index.php/revista/article/view/196/128. Acesso em: 27 set. 202

56. Borges JÁ, et al. Fadiga: Um Sintoma Complexo e seu Impacto no Câncer e na Insuficiência Cardíaca. International Journal of Cardiovascular Sciences, Rio de Janeiro, Brasil, v. 31, ed. 4, p. 433-442, 2018. DOI http://dx.doi.org/10.5935/2359-4802.20180027. Disponivel em: https:/www.scielo.br/pdf/ijcs/v31n4/pt_2359-4802ijcs-31-04-0433.pdf. Acesso em: 30 set. 2020.

57. Watte $G$, et al. Perda de peso proporcional em seis meses como fator de risco para mortalidade no câncer de pulmão de células não pequenas estádio IV. J Bras Pneumol [Internet]. 2018 Apr 22 [cited 2020 Sep 9];44(6):505-509. DOI https:/doi.org/10.1590/s180637562018000000023. Available from: https:/www.scielo.br/pdf/jbpneu/v44n6/pt_1806-3713-jbpneu-44-06-00505.pdf

58. Godoi, LT; Fernandes, SL. Terapia nutricional em pacientes com câncer do aparelho digestivo. International Journal of Nutrology, Rio de Janeiro, Brasil, v. 10, ed. 4, p. 136-144, setembro 2017. DOI 10.1055/s0040-1705645. Disponível em: https:/www.thieme-connect.com/ products/ejournals/abstract/10.1055/s-0040-1705645. Acesso em: 30 set. 2020.

59. Cruz, FCS, et al. O estado nutricional e a alimentação via sonda estão associados à qualidade de vida em pacientes oncológicos em cuidados paliativos?. DEMETRA, alimentação, nutrição e saúde, Minas Gerais, Brasil, v. 14, ed. e38198, p. 1-14, abril 2019. DOI DOI: 10.12957/ demetra.2019.38198. Disponivel em: https:/www.e-publicacoes.uerj. br/index.php/demetra/article/view/38198/29397. Acesso em: 30 set. 2020. 


\section{REFERÊNCIAS}

60. Arends, J. et al. ESPEN guidelines on nutrition in cancer patients. Clinical Nutrition: ELSEVIER, EUROPEAN, v. 36, p. 1-48, 2017. DOI 10.1016/j.clnu.2016.07.015. Disponivel em: https://pubmed.ncbi.nlm. nih.gov/27637832/. Acesso em: 30 set. 2020.

61. Castro JMF, et al. Consensos e dissensos na indicação e continuidade da terapia nutricional enteral nos cuidados paliativos de pacientes com doenças crônicas não transmissíveis. Arquivos Brasileiros de Ciências da Saúde [Internet]. 2017 [cited 2020 Aug 19];42(1):55-59. DOI https:/doi.org/10.7322/abcshs.v42i1.951. Available from: https:/ pesquisa.bvsalud.org/portal/resource/pt/biblio-833100

62. Santos RCC; Brandão GRR; Oliveira, JGP. Perfil nutricional de pacientes portadores de neoplasia do trato gastro intestinal (TGI) antes, durante e após tratamento sistêmico. Brazilian Journal of health Review, Bahia, Brasil, v. 3, ed. 4, p. 9185-9204, Agosto 2020. DOI 10.34119/ bjhrv3n4-158. Disponivel em: https:/www.brazilianjournals.com/index.php/BJHR/article/view/13802/11548. Acesso em: 30 set. 2020

63. Cruz, FCS, et al. O estado nutricional e a alimentação via sonda estão associados à qualidade de vida em pacientes oncológicos em cuidados paliativos?. DEMETRA, alimentação, nutrição e saúde, Minas Gerais, Brasil, v. 14, ed. e38198, p. 1-14, Abril 2019. DOI DOl: 10.12957/ demetra.2019.38198. Disponivel em: https://www.e-publicacoes.uerj. br/index.php/demetra/article/view/38198/29397. Acesso em: 30 set. 2020.

64. Kapoor, N. et al. A Prospective Randomized Controlled Trial to Study the Impact of a Nutrition-Sensitive Intervention on Adult Women With Cancer Cachexia Undergoing Palliative Care in India. Integr Cancer Ther., İndia, v. 16, ed. 01, p. 74-84, 16 mar. 2017. DOI 10.1177 / 1534735416651968. Disponivel em: https:/www.ncbi.nlm.nih.gov/ pmc/articles/PMC5736069/pdf/10.1177_1534735416651968.pdf. Acesso em: 4 out. 2020.

65. Godoi LT; Fernandes, SL. Terapia nutricional em pacientes com câncer do aparelho digestivo. International Journal of Nutrology, Rio de Janeiro, Brasil, v. 10, ed. 4, p. 136-144, Setembro 2017. DOI 10.1055/s0040-1705645. Disponivel em: https://www.thieme-connect.com/ products/ejournals/abstract/10.1055/s-0040-1705645. Acesso em: 30 set. 2020.

66. Peruchi RFP, et al. Suplementação Nutricional em Idosos (Aminoácidos, Proteínas, Pufas, Vitamina D e Zinco) com ênfase em Sarcopenia; Uma revisão sistemática. Revista UNINGÁ, Ribeirão Preto- São Paulo, 25 abr. 2017.

67. Teshima CK; Vasconcelos CMT; Takemoto E. Suplementação de Aminoácidos: Influência na Sarcopenia em Idosos-uma Breve Revisão. Thieme Revinter, Rio de Janeiro, Brasil, v. 11, ed. 1, p. 324-327, 2018. DOI DOl: 10.1055/s-0038-1675030. Disponível em: https:/www.thieme-connect.com/products/ejournals/html/10.1055/s-0038-1675030. Acesso em: 1 out. 2020.

68. Hanna, R. et al. A Practical Approach to Nutrition, Protein-Energy Wasting, Sarcopenia, and Cachexia in Patients with Chronic Kidney Disease. Blood Purification: Blood Purif, University of California Irvine School of Medicine, p. 1-10, 18 dez. 2019. DOI 10.1159 / 000504240. Disponivel em: https:/www .ncbi.nlm.nih.gov/ pubmed/31851983. Acesso em: 28 dez. 2019.

69. Jentoft AJC. Sarcopenia: what should a pharmacist know?. Farmacia
Hospitalaria, Madrid, v. 41, ed. 41, p. 543-549, 5 abr. 2017. DOI DOI: 10.7399/fh.2017.41.4.10802. Disponivel em: https:/www.sefh.es/ fh/161_11revision0110802.pdf. Acesso em: 1 out. 2020.

70. Dolan, E; Gualano B; Rawson, E. Beyond muscle: the effects of creatine supplementation on brain creatine, cognitive processing, and traumatic brain injury. European Journal of Sport Science, European, v. 1, p. 1-16, 7 ago. 2018. DOI 10.1080 / 17461391.2018.1500644. Disponivel em: https:/www.ncbi.nlm.nih.gov/pubmed/30086660. Acesso em: 28 dez. 2019.

71. Teixeira Y. et al. Efeitos da Suplementação de Creatina Sobre o Desempenho Físico: Uma revisão integrativa de literatura. Health Sciences, Juazeiro do Norte, v. 9, ed. 7, p. 1-7, 2020. DOI http:/dx.doi. org/10.33448/rsd-v9i7.4947. Disponivel em: https:/www.rsdjournal. org/index.php/rsd/article/view/4947. Acesso em: 1 out. 2020.

72. Jentoft AJC, Sarcopenia: what should a pharmacist know?. Farmacia Hospitalaria, Madrid, v. 41, ed. 41, p. 543-549, 5 abr. 2017. DOI DOI: 10.7399/fh.2017.41.4.10802. Disponivel em: https:/www.sefh.es/ fh/161_11revision0110802.pdf. Acesso em: 1 out. 2020.

73. Gago LC; Gago, FC. Atualidades sobre o tratamento da Sarcopenia revisão de literatura. International Journal of Nutrology, Rio de Janeiro, Brasil, v. 9, ed. 4, p. 254-271, 2016. DOI 10.1055/s-0040-1705640. Disponivel em: https://www.thieme-connect.com/products/ejournals/ abstract/10.1055/s-0040-1705640. Acesso em: 1 out. 2020.

74. Mats N. et al. A Five-Ingredient Nutritional Supplement and Home-Based Resistance Exercise Improve Lean Mass and Strength in Free-Living Elderly. Nutrients., Canadá, v. 12, ed. 2391, p. 1-28, 3 ago. 2020. DOI doi: 10.3390 / nu12082391. Disponível em: https://pubmed. ncbi.nlm.nih.gov/32785021/. Acesso em: 4 out. 2020.

75. Avgerinos, K. I. et al. Effects of creatine supplementation on cognitive function of healthy individuals: A systematic review of randomized controlled trials. Experimental Gerontology, [s.l.], v. 108, p.166-173, jul. 2018. Elsevier BV.

76. Yooh S, et al. Effects of Creatine Monohydrate Augmentation on Brain Metabolic and Network Outcome Measures in Women With Major Depressive Disorder. Biological Psychiatry [Internet]. 2016 Sep 15 [cited 2020 Sep 8];80(6):1-9. DOI https://doi.org/10.1016/j.biopsych.2015.11.027. Available from: https:/www.sciencedirect.com/ science/article/abs/pii/S0006322315010422

77. Benedetto $F$, et al. Supplementation with Qter ${ }^{\circledR}$ and Creatine improves functional performance in COPD patients on long term oxygen therapy. Respiratory Medicine: ELSEVIER, ITALY, v. 142, p. 89-93, 1 ago. 2018. DOI 10.1016 / j.rmed.2018.08.002. Disponivel em: https:/www.resmedjournal.com/action/showPdf?pii =S0954-6111\%2818\%2930259-2. Acesso em: 4 out. 2020.

78. Fairman C, et al. Examining the effects of creatine supplementation in augmenting adaptations to resistance training in patients with prostate cancer undergoing androgen deprivation therapy: a randomised, double-blind, placebo-controlled trial. BM] OPEN [Internet]. 2019 Sep 20 [cited 2020 Oct 1];9(9):1-9. DOI 10.1136 / bmjopen-2019-030080. Available from: https:/www.ncbi.nlm.nih.gov/pmc/ articles/PMC6756416/. 